\title{
STRUCTURE AND BEHAVIOR OF RAT PRIMARY AND SECONDARY SCHWANN CELLS IN VITRO
}

\author{
M. DUBOIS-DALCQ, B. RENTIER, A. BARON, N. van EVERCOOREN and B. W. BURGE
}

Infectious Diseases Branch, National Institute of Neurological and Communicative Disorders and Stroke, National Institutes of Health, Bethesda, MD 20205, USA

\begin{abstract}
SUMMARY
The structure and motility of isolated rat primary (I) Schwann cells (SC) have been compared to that of subcultured (II) SC during and after mitotic stimulation. I SC contain myelin components which persist for 2 weeks in serum-free medium while they rapidly disappear in medium containing serum and high glucose concentration. These components were never detected in II SC. Both I SC and II SC after their mitotic phase are spindle-shaped, contain many intermediate and actin filaments, have no basement membrane but show intense migratory and undulatory activities. Rare fibroblasts in I cultures are recognized by their extremely variable shape, the presence of Thy 1.1 antigen in their membrane and their intense edge ruffling alternating with abrupt translocation. In contrast, I SC movements consist of intracellular translocation of nuclei along SC processes, which retract and extend constantly, and in slow rhythmic undulation episodes $(2.3 \pm 0.2 / \mathrm{min})$ alternating with migration at $135 \pm 50 \mu / \mathrm{h}$. The total number of these episodes per day in serum-free medium is rigorously identical for different cells (166.3 \pm 0.2$)$ and this uniformity of frequency suggests a genotypic basis. Cycles, consisting of an undulation episode followed by a resting interval, have mean durations of $8.6 \pm 4.1 \mathrm{~min}$ and a sharp peak of occurrence at $6 \mathrm{~min}$, with exponential distribution of the longer periods. Motility of II SC is considerably inhibited during mitotic stimulation by cholera toxin and a pituitary extract while SC phenotype has changed to a flat multipolar cell with prominent Golgi and ribosomes. Migration is reduced to $24 \pm 2 \mu / \mathrm{h}$ and only $2 \%$ of the SC show pulsations of the same periodicity as the I SC undulations. A dramatic increase in pulsation frequency occurs 6-12 h after removal of mitogenic factors when $80 \%$ of II SC start pulsating twice as fast for 2 3 days. When mitoses cease, SC quickly recover their SC phenotype with rhythmic undulations while migration speed increased to $92 \pm 20 \mu / \mathrm{h}$. Thus, in spite of dramatic modification of shape, structure and behavior during mitotic stimulation, SC subsequently recover their unique motility pattern which might be essential for their myelinating function.
\end{abstract}

Rat Schwann cells (SC), the myelin-form-ing cells of the peripheral nervous system, can now be cultured in isolation by using two different techniques [1-3]. In the first, rat dorsal root ganglia obtained just before birth are explanted and treated with antimitotic agents for a few days. After 10 days, the ganglia are excised and a "Schwann cell bed" is developed after the neurites are eliminated [1,2]. In the second technique, dissociation of newborn rat sciatic nerve results in a mixed population of SC and fibroblasts, which after 2 days of culturing are identified by their respective surface antigenic markers Ran 1 and Thy 1.1 [3, 4]. In these cultures, fibroblast growth is first inhibited by AraC treatment and subsequently, by complement-mediated killing of Thy 1.1 positive cells [3]. SC growth can then be intensely stimulated by pituitary extract, cholera toxin, or both factors together in the presence of fetal calf serum (FCS) [5]. The advantage of the second technique is that large quantities of SC can be obtained by subculturing. It is now possible therefore, to study the behavior, structure and function of SC cultured in isolation or in the presence of other cell types such as neurons.

In the present study, SC have been cultured in various media which influence expression of myelin-specific components in these cells $[6,7]$. Primary and secondary SC were studied by phase-contrast video-intensification microscopy (VIM) [8], scanning (SEM) and transmission electron microscopy (TEM). These revealed characteristic differences in motility and structure between SC and fibroblasts as well as between primary and secondary SC under mitotic stimulation. However, after mito-genic factors had been eliminated for several days, secondary SC stopped dividing and recovered their original primary SC behavior and structure.

\section{MATERIALS AND METHODS}

Culturing techniques [3]

Primary cells. Briefly, sciatic nerves from newborn rats 2-3 days old (Fisher, Sprague-Dawley or Osborn Mendel) were excised and dissociated with trypsin and collagenase type III (Worthington Biochemical Corp., Freehold, N.J.) followed by passage through a no. 23 hypodermic needle. This usually resulted in a single cell suspension, negating the need for further filtration. Thirtyfive $\mathrm{mm}$ Petri dishes were seeded with $6 \times 10^{5}$ cells for 
use in TEM and VIM studies (see below). Thirteen mm glass coverslips were seeded with $5 \times 10^{4}-10^{5}$ cells for immunofluorescence, autoradiography and SEM. The three media most often used were: (a) Dulbecco's Modified Eagle Medium (DMEM) with 10\% fetal calf serum (FCS) from Microbiological Associates (Walkersville, Md); (b) "Nerve Cell" MEM [9] which is MEM-enriched in sodium bicarbonate and glucose (600 mg \%) supplemented with FC or horse serum (Gibco, Grand Island, NY); (c) serum-free DMEM supplemented with bovine insulin, transferrin, progesterone, putrescine, and sodium selenate at the concentrations described [10] (all products from Sigma except selenium, United Mineral and Chemical Corp., New York, NY). Primary cells were always grown in serum for the first $48 \mathrm{~h}$. Subsequently, in some cases, cytosine-arabino-side (AraC) was diluted in the serum-containing medium at $10^{-5} \mathrm{M}$ and maintained on the cells for 2 days. AraC was then carefully washed off and replaced by dibutyryl cyclic AMP (db-cAMP) also at $10^{-5} \mathrm{M}[11]$. In other cases, AraC treatment was not carried out and serum-free medium was substituted for serum-containing medium after 2 days (db-cAMP was added as above).

Secondary cells. Secondary cells were established as described [3]. Briefly, cells were removed from the dishes by trypsin-EDTA $\left(15 \mathrm{~min}, 37^{\circ} \mathrm{C}\right)$ and reseeded without further treatment when only a few fibroblasts were present. If more fibroblasts were identified, trypsinized cells were treated with a dilution (1:200) of anti-Thy 1.1 monoclonal IgM antibody (New England Nuclear, Boston, MA) and freshly thawed rabbit complement. Secondary cells were plated in $35 \mathrm{~mm}$ Petri dishes or $25 \mathrm{~cm}^{2}$ Falcon flasks and, when larger number of cells were obtained, in $75 \mathrm{~cm}^{2}$ flasks. These cells were always grown in DMEM with $10 \% \mathrm{FC}$ serum and supplemented with bovine pituitary extract (see below) at $12.5 \mu \mathrm{g} / \mathrm{ml}$. In addition, cholera toxin from Vibrio cholerae, (Sigma Chemical Co., St Louis, MO) was often added at $1 \mu \mathrm{g} / \mathrm{ml}$ [5], Tissue extracts and cholera toxin have such synergistic and stimulating effects on SC that doubling occurs in 2-3 days.

\section{Pituitary growth factor}

Pituitary growth factor was prepared as described [3, 5] with additional purification stages [12]. After the first purification by precipitation in $\left(\mathrm{NH}_{4}\right)_{2} \mathrm{SO}_{4}$ at $\mathrm{pH} 4.5$, the supernatant was adjusted to $\mathrm{pH}$ 6.5-7.0 and treated twice with powdered $\left(\mathrm{NH}_{4}\right)_{2} \mathrm{SO}_{4}$. The pellet was then suspended in a minimal volume of $0.1 \mathrm{M}$ phosphate buffer, $\mathrm{pH}$ 6.0, and dialysed extensively before being filtered through CM52 carboxy-methylcellulose (Whatman Biochemicals, Maidstone, Ky) equilibrated with the same buffer (batch procedure). The CM52 was washed with $0.1 \mathrm{M}$ phosphate and $0.05 \mathrm{M} \mathrm{NaCl}$. Elution was then performed with $0.1 \mathrm{M}$ phosphate buffer and $0.2 \mathrm{M} \mathrm{NaCl}$. One hundred lyophilized pituitary glands (bovine, anterior lobes, from Pel-Freeze, Rogers, Ak) generally yielded $\pm 15 \mathrm{mg}$ of growth factor.

\section{Video-intensification microscopy (VIM)}

Cultures were observed using time-lapse VIM equipment as developed by Willingham \& Pas tan [8]. Briefly, cells grown in $35 \mathrm{~mm}$ plastic Petri dishes or $25 \mathrm{~cm}^{2}$ Falcon flasks were placed on the specimen stage of a Zeiss Invertoscope $\mathrm{D}$ partially enclosed in a plexiglass box. Atmosphere inside the box was maintained at $37^{\circ} \mathrm{C}$ by a YSI 72 proportional temperature controller combined with an air circulator and constantly supplied with $10 \%$ $\mathrm{CO}_{2}$. Illumination was maintained at a minimum level $(1.2 \mathrm{~V})$ in order to avoid light-induced damage to the cells. The microscope was equipped with a Zeiss Plan 40 phase contrast objective featuring a variable numeric aperture (1.1-1.5). Image was collected by an RCA silicon-intensifier target camera, the sensitivity of which is $10^{6}$ times greater than standard black and white video cameras. Signal from the camera was transmitted to a Panasonic NV-8030 time-lapse 1/2 inch video tape recorder and to a Hitachi video monitor. Recordings were made by taking individual frames at $1.8 \mathrm{sec}$ intervals. This permitted observation of movements with an acceleration of 108 times their real speed. Faster playback was obtained by copying the original tape with a second recorder, increasing 9 times the playback speed (972 times final).

\section{Immunofluorescence and autoradiography [3, 6, 13]}

For immunofluorescence, living cells on glass cover-slips were stained with monoclonal anti-Thy 1.1 IgM kindly provided by Dr M. Raff or from New England Nuclear (see above). Acetone-fixed cells were stained with two different rabbit anti- $\mathrm{P}_{0}$, glycoprotein sera. One serum directed against rat peripheral nerve Po was kindly provided by Dr J. Brockes [6, 7]. The other serum, directed against bovine spinal root Po, was a gift from Dr B. Trapp [14]. Protein A-fluo-rescein (Pharmacia Fine Chemicals, Inc., Piscataway, N.J.) was used as the second layer.

For autoradiography [13], cells were incubated $24 \mathrm{~h}$ in medium containing $6 \mu \mathrm{Ci} / \mathrm{ml}\left[{ }^{3} \mathrm{H}\right]$ thymidine (New England Nuclear, $46.8 \mathrm{Ci} / \mathrm{mole})$. SC were then washed and fixed in acid-alcohol, rinsed, dried and covered with Kodak NTB2 emulsion. After 1 day, they were developed with Dektol and fixed before being counter-stained with cresyl violet. 


\section{Electron microscopy}

Glass coverslips were fixed, dehydrated, critical point-dried and gold-coated as described [9]. Coverslips were examined in an ETEC autoscan at a tilt angle of $45^{\circ}$. Pellets of cells were fixed in Karnovsky's fixative and embedded in conical capsules while Petri dishes were fixed in situ with Karnovsky's fixative and embedded as described [9]. Groups of SC were circled, drilled out and remounted for thin sectioning. Grids were counterstained with uranyl acetate and lead citrate and observed in a Philips 201 TEM.

Fig. 1. Thin cross-section of a Schwann cell (SC) pellet resulting from the dissociation of newborn rat sciatic nerve. Cells contain folded myelin sheaths showing typical periodicity (inset) surrounding a swollen axoplasm or an empty space. x5800; inset, x 29000.

Fig. 2. Differences in phase contrast morphology between SC maintained 5 days in $(a)$ serum-containing medium; $(b)$ in serum-free medium. Primary cells have a typical spindle shape and long processes which are especially thin in serum-free medium. In contrast, rare fibroblasts (F) are very flat and fibrillar. Small granules or vacuoles (arrows) can be detected in the SC perinuclear cytoplasm which is scarce in cells maintained in serum-free medium. x368.

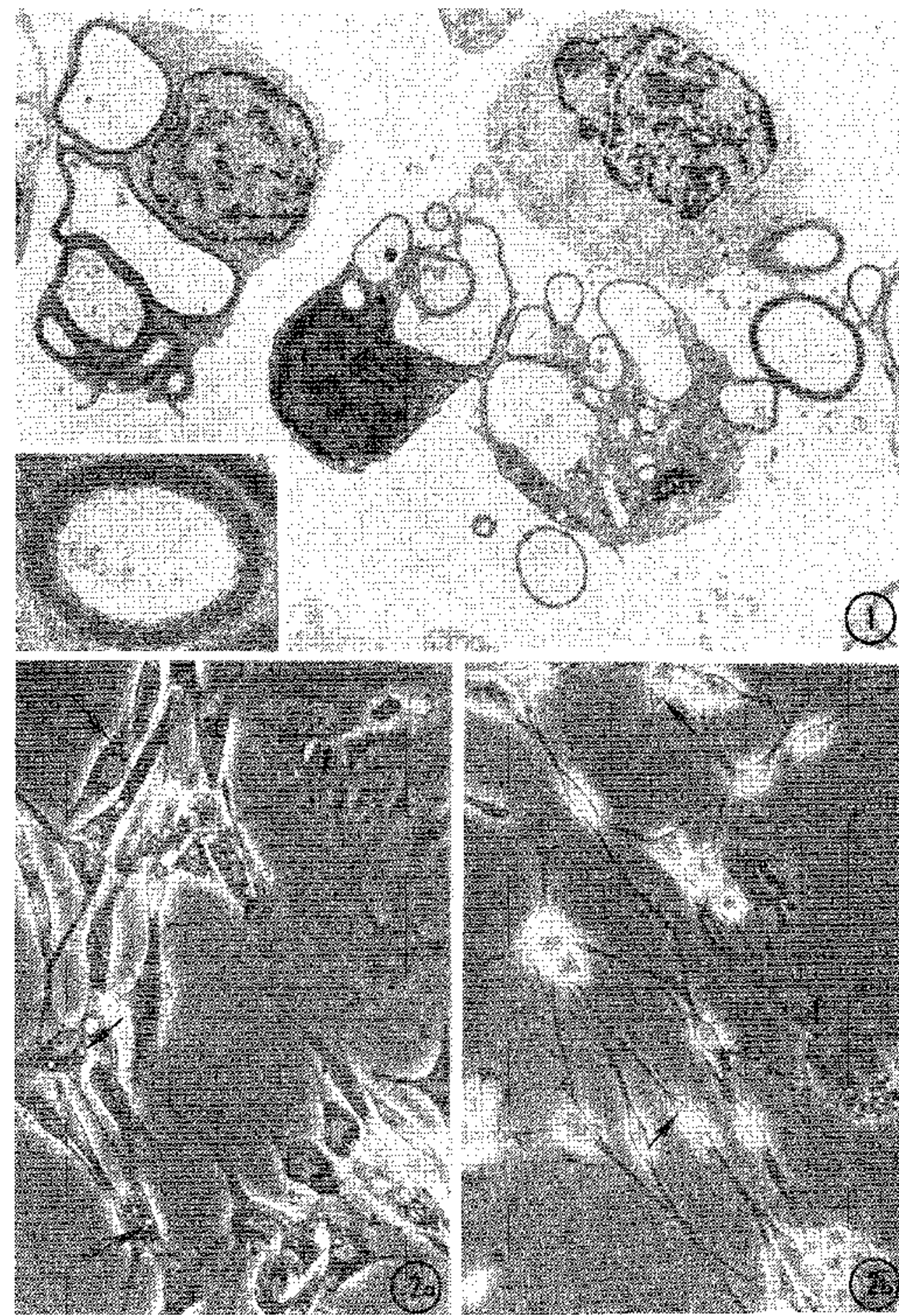


RESULTS Primary Schwann cells SC obtained as the result of sciatic nerve dissociation contained myelin sheaths as revealed by TEM (fig. 1). This myelin surrounded axons which showed acute swelling and dissolution of neurotubules as one would expect to see following multiple transections. Myelin tubes had lost their normal rigidity and had folded in the course of dissociation so that the normal ratio of one myelinated fiber to one SC [15] was apparently lost (fig. 1). Other putative SC showed no myelin but rather several large cytoplasmic vacuoles which probably contained unmyelinated axons before dissociation. None of these SC had basement membrane or collagen around them, as expected after enzymatic treatment.

When SC were plated, they rapidly acquired a spindle shape and two permanent bipolar processes. Occasional thin lateral processes were observed. Fibroblast growth was inhibited by treatment with AraC or by switching to serum-free medium after 2 days in culture. After 5-7 days, SC grown in serum-free medium appeared thinner than those cultured in serum-containing medium and rarely formed groups (fig. $2 a, b$ ). Mitoses were rare in the stable cultures and primary SC monolayers never became confluent as did secondary ones. Phase-bright granules could sometimes be detected close to the SC nucleus in the phase microscope (fig. 2, arrows) and, with TEM, numerous inclusions of intact or partially digested myelin were detected in the cell body even after 2 weeks in serum-free medium (fig. 3). This observation correlated well with the presence of perinuclear immunofluorescent granules seen after staining with anti- $\mathrm{P}_{0}$ glycoprotein serum [6,7] (fig. 3, inset). In contrast, SC cultured in "Nerve Cell" MEM and serum (after AraC treatment) showed no $\mathrm{P}_{0}$ staining or myelin inclusions after 7 days (fig. 4). Sometimes, myelin inclusions were not found after 2 days in this medium as if its high glucose concentration had triggered rapid digestion of the myelin present right after dissociation. The cells showed well developed Golgi in the perinuclear region, scattered rough ER, and free ribosomes intermixed with filaments and occasional dense bodies as seen in rat SC obtained by successive reexplantations [16]. In no case were basement membrane and collagen seen to reappear during culturing, in agreement with previous observations $[17,18]$.

Figs 3,4. Ultrastructural aspect of primary SC cultured in serum-free medium (fig. 3) and in serum-containing medium with high glucose (fig. 4). Cells in serum-free medium contain many myelin inclusions at 7 days as seen in fig. 3. Myelin periodicity has been altered except at arrow. These cells also contain im-munofluorescent granules after staining with anti- $\mathrm{P}_{0}$ glycoprotein antiserum (inset). In contrast, in fig. 4 , SC cytoplasm and processes are completely devoid of myelin inclusions. Note prominent Golgi apparatus on the left and abundant bundles of filaments intermixed with dense bodies (arrows) and rough ER. Fig. 3, x47000; inset, x550; fig. 4, x9000.

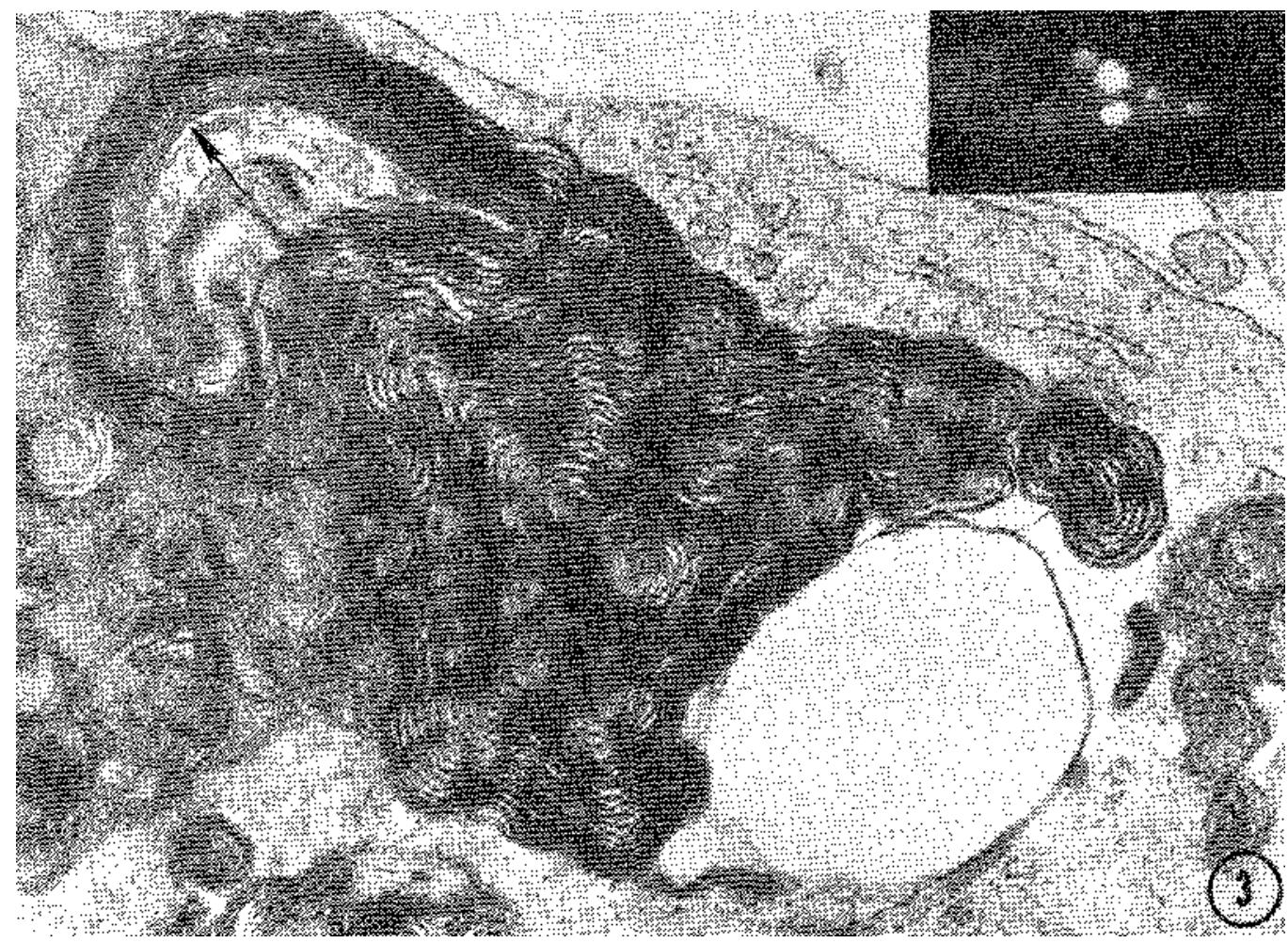




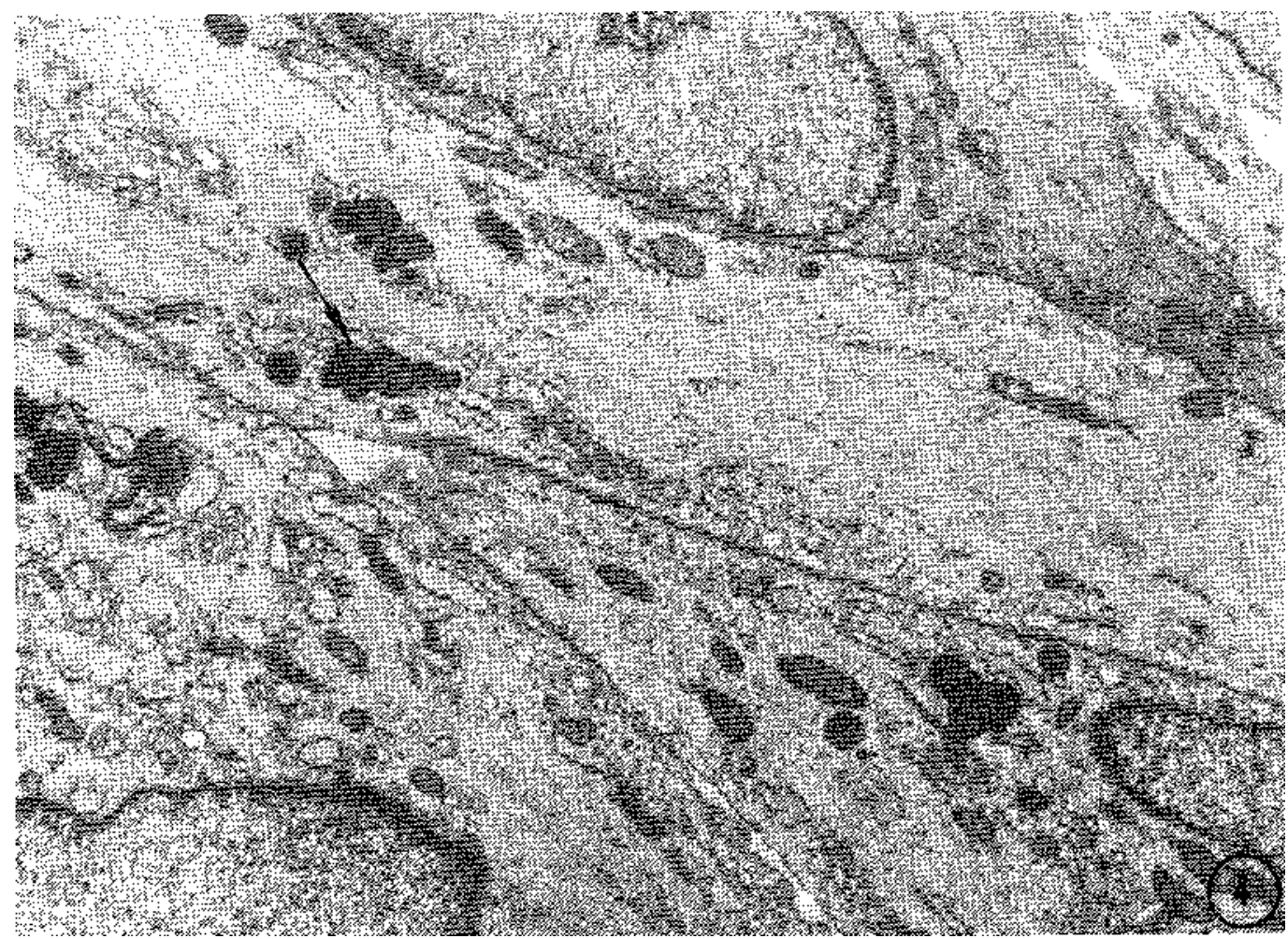

The differences in motility and structure between SC and the occasional fibroblasts present in primary cultures were studied by VIM and TEM. Fibroblasts were polymorphic and had intense edge ruffling. They were essentially static but sometimes migrated as described [19]. They crawled across the observation field at 160 $( \pm 10) \mu / \mathrm{h}$ in an apparently random fashion. Occasionally, rapid translocation of fibroblasts occurred over short distances, ending in settlement under a group of SC. These events were correlated with the extremely polymorphic shape and size displayed by Thy 1.1 positive cells after immunofiuorescent staining. Some positive cells were polygonal, or triangular and flat while others were apparently retracted and had multiple and tortuous extensions (fig. 5 inset). SEM and TEM revealed that such cells, probably caught by the fixative in the middle of a migration process, had bundles of packed filaments localized along the convex side of the cell, acting as a backbone (figs 5,6). These cells showed extensive and dilated ER, convoluted nuclei and large surface blebs unlike SC.

Primary SC had characteristic motility as described before [20-23] and their processes contained numerous filaments identified as actin ( $6 \mathrm{~nm}$ in diameter) and intermediate filaments (9-10 nm in diameter) (fig. 4). Some stages of these movements were caught by fixation and examined with SEM (figs 6, 7). The movements consisted of $(a)$ rhythmic slow undulation of the cell body (or side-to-side movement); $(b)$ migration from one group to another by sliding along the length of each other (fig. 6); (c) intracellular translocation of nuclei along SC processes; $(d)$ extension and retraction of processes with fine ruffling at the triangular extremities (fig. 7).

Some of the SC movements were studied by VIM in greater detail. The undulating movement consists of a strong contraction displacing the cell body to one side, followed by 3 or 4 side-to-side undulations decreasing in amplitude, and a resting interval (fig. 8). The duration of the undulation episodes was rather constant (2.3 \pm 0.2 min) but the resting interval was quite variable. As a result, the duration of the whole cycle (undulation episode plus interval) varied greatly, ranging from 2.34 to $44.46 \mathrm{~min}$ (mean=8.66 $\pm 4.81 \mathrm{~min}$ ). A sharp peak of frequency occurred at approx. $6 \mathrm{~min}$, with an exponential distribution of the longer periods (fig. 9). When measured over long periods of time, the total number of cycles/ day was remarkably identical for different cells in the same culture fed with serum-free medium $(166.3 \pm 0.2$ cycles/24 h). The migratory movement of SC occurred discontinuously and often during the non-undulating phases. Migration activity of SC started after the cells had settled on the substrate and had become spindle-shaped. The path of migration was often oriented along a constant axis for every cell, in contrast with the random migratory path of fibroblasts. Back and forth motion of SC along their axis was often observed. From 6 to $18 \mathrm{~h}$, migration speed increased from $55 \pm 45 \mu, / \mathrm{h}$ to $140 \pm 40 \mu / \mathrm{h}$. SC maintained the same speed during the following days and at 28 days were still averaging $120 \mu / \mathrm{h}$ (fig. 10). Although the speed of primary SC was quite variable as shown by the standard deviation values in fig. 10, the cells were constantly moving and groups often seen in the culture were only transient. 
Fig. 5. TEM of a cell identified as a fibroblast migrating between SC. Numerous blebs $(b)$ are present along the plasma membrane on the left. Note also a prominent filament bundle extending the length of the cell background. Endoplasmic reticulum cisternae are numerous and dilated. Inset shows immunofiuorescent staining of Thy 1.1 antigen on the surface of such a migrating fibroblast. x5 400 , inset, x470.

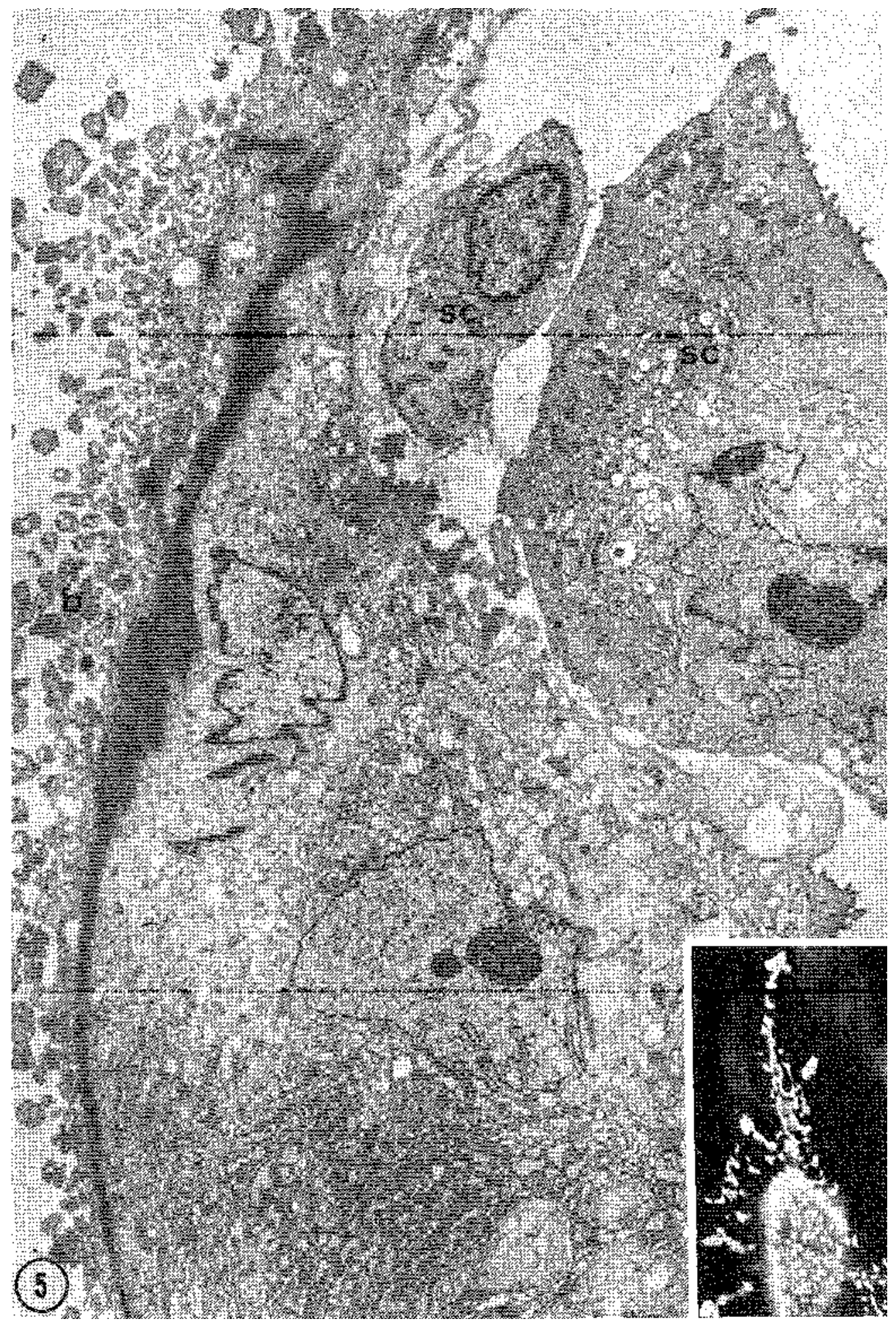

\section{Secondary cells}

In early subcultures, the phenotype of cells grown in medium-containing pituitary factor (PITF) was different from the cells grown in PITF and cholera toxin (CT). PITF-stimulated cells were very elongated and bipolar while PITF and CT-stimulated cells, as well as CT-stimulated SC, were flatter, triangular and often multipolar (fig. 1la) [24]. Immediately after plating, secondary SC extended bipolar processes which ended in a ruffling expansion usually longer than those of primary SC. This resulted in temporary contact with other cells or cell 
processes. After 5-8 h, additional processes grew, resulting in multipolar cells which formed a network. Multipolarity and cell flattening, referred to as "CT phenotype", contributed to monolayer conflu-ency within the first $24 \mathrm{~h}$ even though no mitosis had been detected by VIM. During the next days of culture in the presence of both mitogenic factors, VIM and autoradiography revealed intense mitotic activity causing an increase in the numer of cells/ surface unit.

Secondary SC were much less motile than primary SC. No side-to-side undulation could be seen as in primary SC but approx. $2 \%$ of the cells showed series of slow rhythmic pulsations for variable short periods of time. Pulsation was composed of extension and retraction of a radial cytoplasmic wave (fig. 8). Periodicity of pulsation ranged from 6.32 to $7.45 \mathrm{~min}$ (mean= $7.02 \pm 0.48 \mathrm{~min})$, with a frequency of $8.55( \pm 1.17)$ pulsations $/ \mathrm{h}$, close to that of undulation cycles in primary SC. Migration speed was $125 \pm 22 \mu / \mathrm{h}$ after $12 \mathrm{~h}$ in culture when cells were still spindle-shaped and scattered. It decreased to $24( \pm 2) \mu / h$ when cells were spread out and confluent (fig. 10). At that time migration occurred along the same orientation axis for all cells in the same microscopic field.

After a $24 \mathrm{~h}$ tritiated thymidine pulse, autoradiography revealed that $59 \%$ of the cells labeled from day 2 to day 3 and $37 \%$ from day 3 to day 4 . Even when mitotic factors were washed from SC after one day, mitosis went on for 2 or 3 days before stopping. VIM revealed that during mitosis SC maintained their extensions but became thinner while their cell body contracted and rounded up. Metaphase and anaphase were clearly identified. Subsequently, the divided cell bodies slid apart along the processes, and daughter cells separated and spread out without visible blebbing. This movement increased SC speed to $95 \mu / \mathrm{h}$ (fig. 10). In contrast to SC, fibroblasts retracted their flat surface into 1 or 2 processes before completely rounding up and going through the various stages of mitosis. The resulting daughter cells showed intense ruffling and blebbing before spreading out and flattening again on the substrate.

SEM of confluent monolayers showed triangular or irregularly shaped flat cells with a bulging nucleus and edges touching each other. By TEM, secondary SC under mitotic stimulation showed no myelin inclusions, no basement membrane and had prominent Golgi apparatus in the perinuclear area. Rough ER was well developed and numerous ribosomes, free, or in rosettes or chains, were present in between scattered filaments.

After mitotic stimulation, SC were maintained for several days in serum-containing medium and became stationary. During that time SC morphology progressively returned to a typical spindle-shape (fig. $11 b$ ). In that process, the large flat surface areas seen in phase contrast and SEM during mitotic stimulation retracted, and left uncovered some substrate between cells (fig. 12). This disrupted the impression of confluency observed earlier and rendered secondary SC very similar to primary SC monolayers. These spindle-shaped cells contained as many bundles of filaments as did primary SC. These filaments consisted of actin and intermediate filaments as in primary SC. Deprivation of growth factors resulted in a striking increase in the frequency of the slow rhythmic cell pulsations, the duration of the pulsatile phase and the number of pulsatile cells (fig. 8). More than $80 \%$ of the cells entered at least one pulsatile phase during $80 \mathrm{~h}$ of observation. The pulsation periodicity ranged from 2.97 to $4.31 \mathrm{~min}$ (mean $=3.77 \pm 0.33 \mathrm{~min})$ with a frequency of $15.91( \pm 0.08)$ pulsations $/ \mathrm{h}$, which is twice the frequency of undulation cycles in primary SC. When the cells became elongated, undulatory movement was progressively recovered having the same periodicity as in primary SC. Migratory speed increased and reached 90 $\mu / \mathrm{h}$ after 4 days (fig. 10).

The behavior of SC was not significantly modified after freezing cells in dimethyl-sulfoxide by standard methods and subsequently thawing them in medium with mitotic factors. Similarly, the number of contaminating fibroblasts was not increased by this manipulation (less than $1 \%$ ). Therefore, it became possible to build up banks of millions of frozen SC and use them as needed. At the present time, secondary SC have been grown through 13 passages. In some cases, a second Thy 1.1 killing was performed at either P5-P6 or P9-P10 because the proportion of fibroblasts, as detected by immunofluorescence, had risen to approx. $5 \%$. Some of the resulting flasks of cultured cells were then completely devoid of fibroblasts and called "pure" SC. 
Figs 6, 7. SEM of primary SC cultures grown in serum-containing medium for 2 (fig. 6 ) to 7 days (fig. 7). In fig. 6 there is a flat and irregularly shaped fibroblast on the left (F). The three SC in the center are aligned along one axis and appear to slide along each other's length. These cells have some small lateral processes with triangular extremities (arrow). In fig. 7, the extremity of an SC process is in contact with a neighbor cell and shows narrow exploratory extensions. Fig. 6, xl 800; fig. 7, x5625.
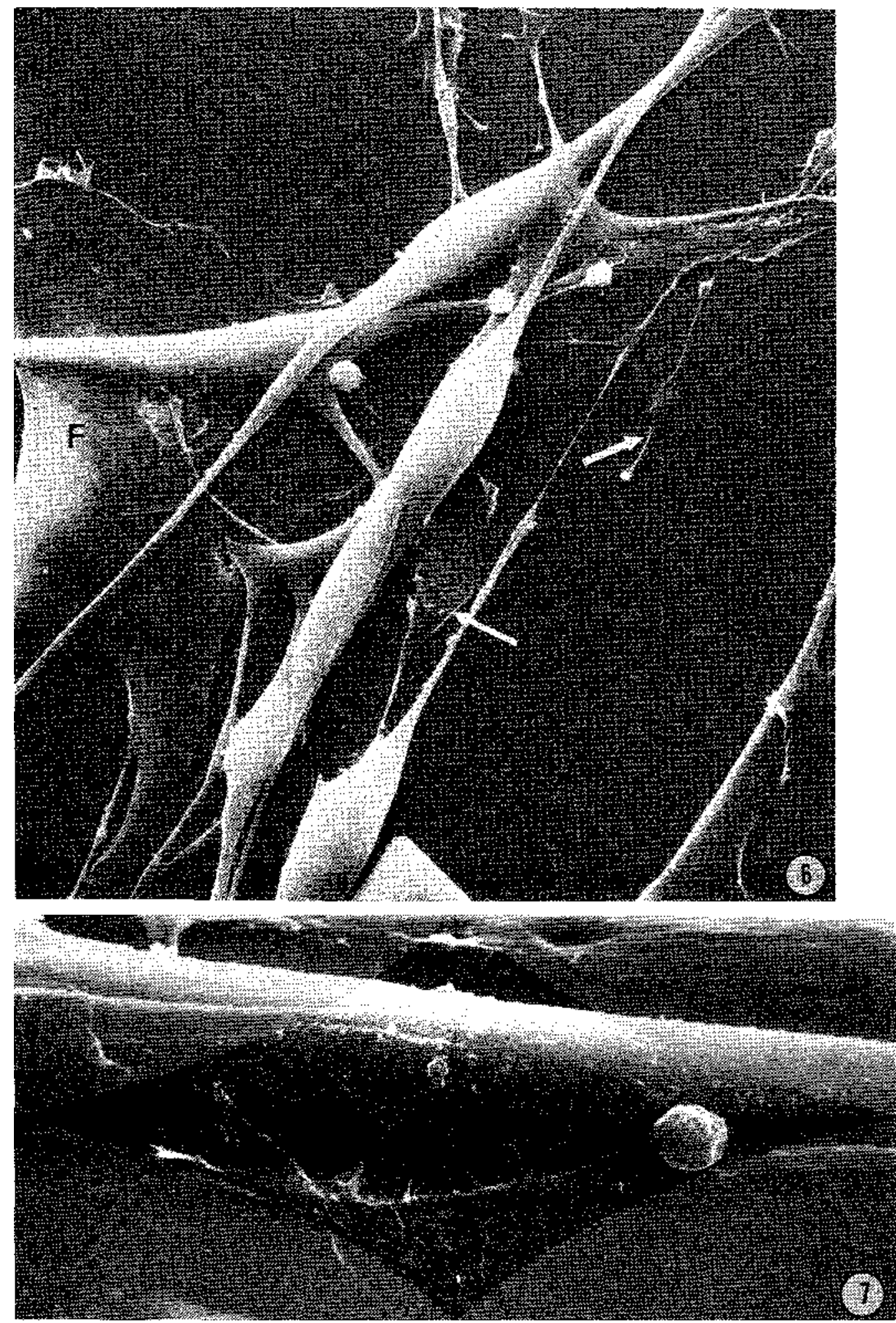
Fig. 8. Schematic representation of SC undulatory and pulsatile activities. Undulations of primary SC (I) occur in cycles composed of an episode of side-to-side movements $(U)$, followed by a resting interval $(R)$. $U$ is relatively constant in duration while $R$ is extremely variable. Pulsatory activity displayed by secondary SC in the presence of mitogenic factors (II A) is schematized. A pulsatory cycle has approximately the same duration as an average undulating cycle in primary SC and is composed of a contraction $(C)$ followed by an extension $(E)$ of the cell body. Only $2 \%$ of the secondary SC pulsate. Pulsation frequency of secondary SC after removal of mitogenic factors (IIB) is twice that shown in II A. More than $80 \%$ of secondary SC display such pulsations. Three days after removal of mitogenic factors, undulations identical to I are recovered.

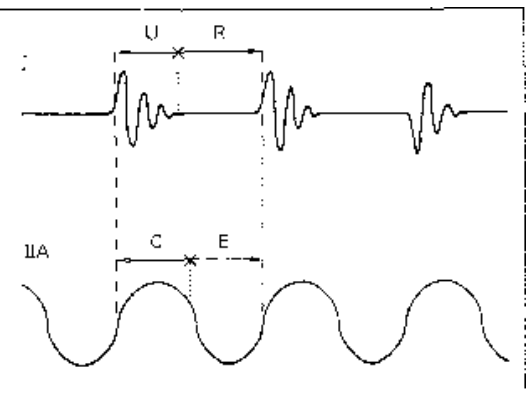

LE

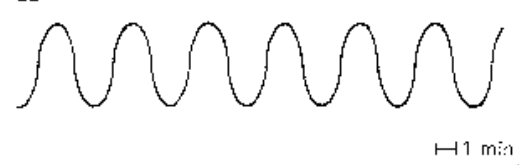

Fig. 9. Distribution of primary SC undulating cycle durations expressed in minutes. Histogram was obtained by cumulative measurement of 338 cycles of $3 \mathrm{SC}$ in synthetic medium during $18 \mathrm{~h}$. Logarithmic processing of the data (inset) demonstrates the exponential distribution of the cycle durations over $5.5 \mathrm{~min}$.

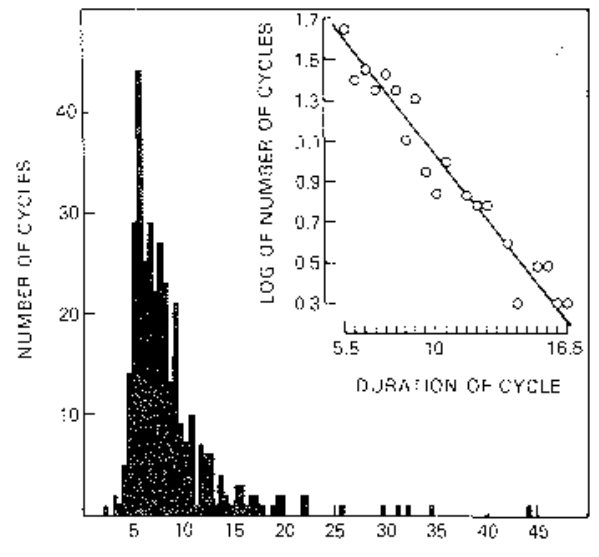

DUFATION OF CYYCEE IU I ? 
Fig. 10. Migratory activity of primary and secondary SC measured by video intensification microscopy. Measurements were made from recording excerpts 7-24 h long. Eight to 14 cells were followed in every culture and 1-4 different cultures were studied in every condition. O, Primary and secondary SC in serum-containing medium; , primary SC in serum-free medium; $\bullet$ SC undergoing mitosis during recording; . fibroblasts. Bottom diagrams indicate the number of mitoses occurring in the video field during recording.
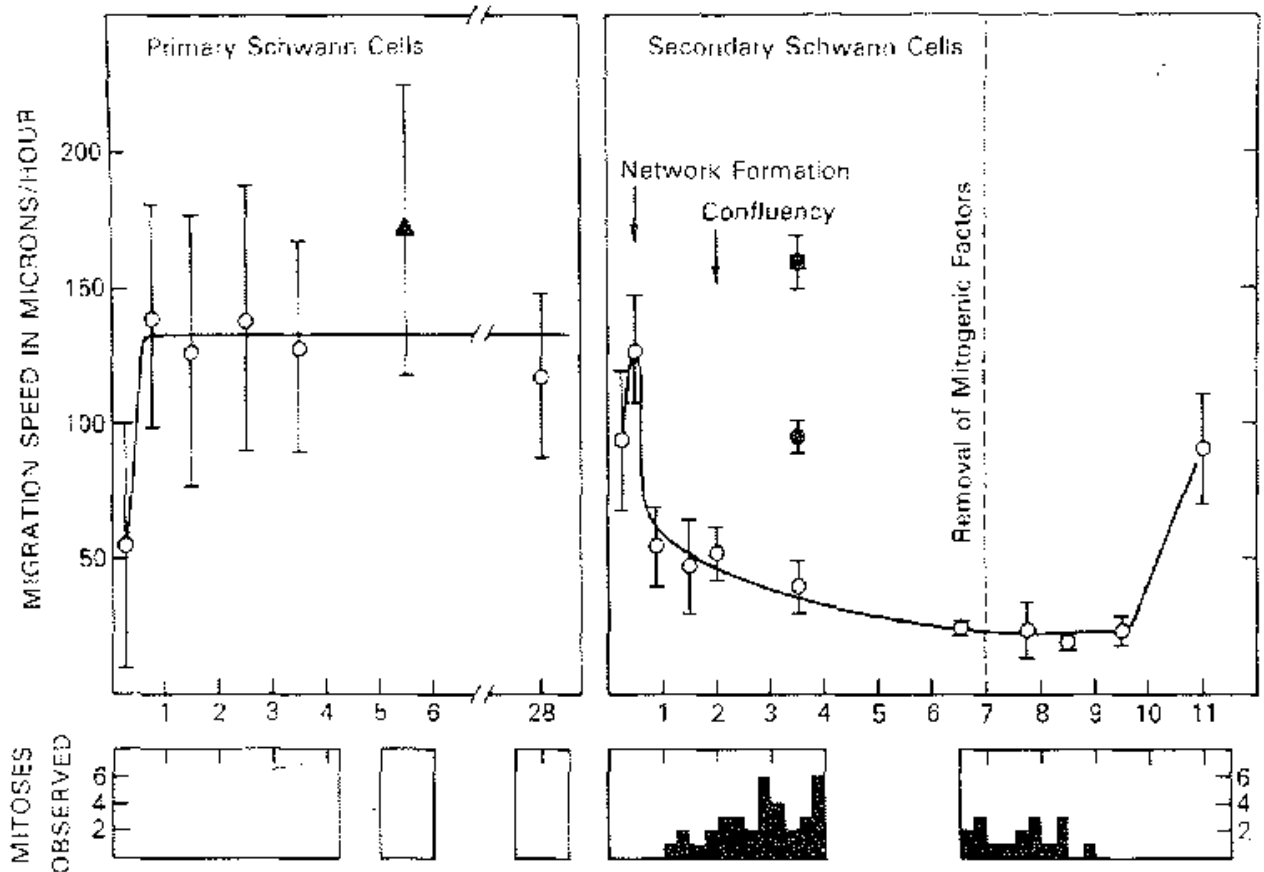

DAYS IN CULTUFF

Fig. 11. Phase contrast morphology of secondary SC during (a) and after (b) mitotic stimulation. In (a) cells are flat, multipolar, and confluent and these aspects are specifically induced by cholera toxin. In (b) cholera toxin and pituitary factor have been removed for 6 days and SC have recovered their spindle shape and non-confluent aspect. x307.
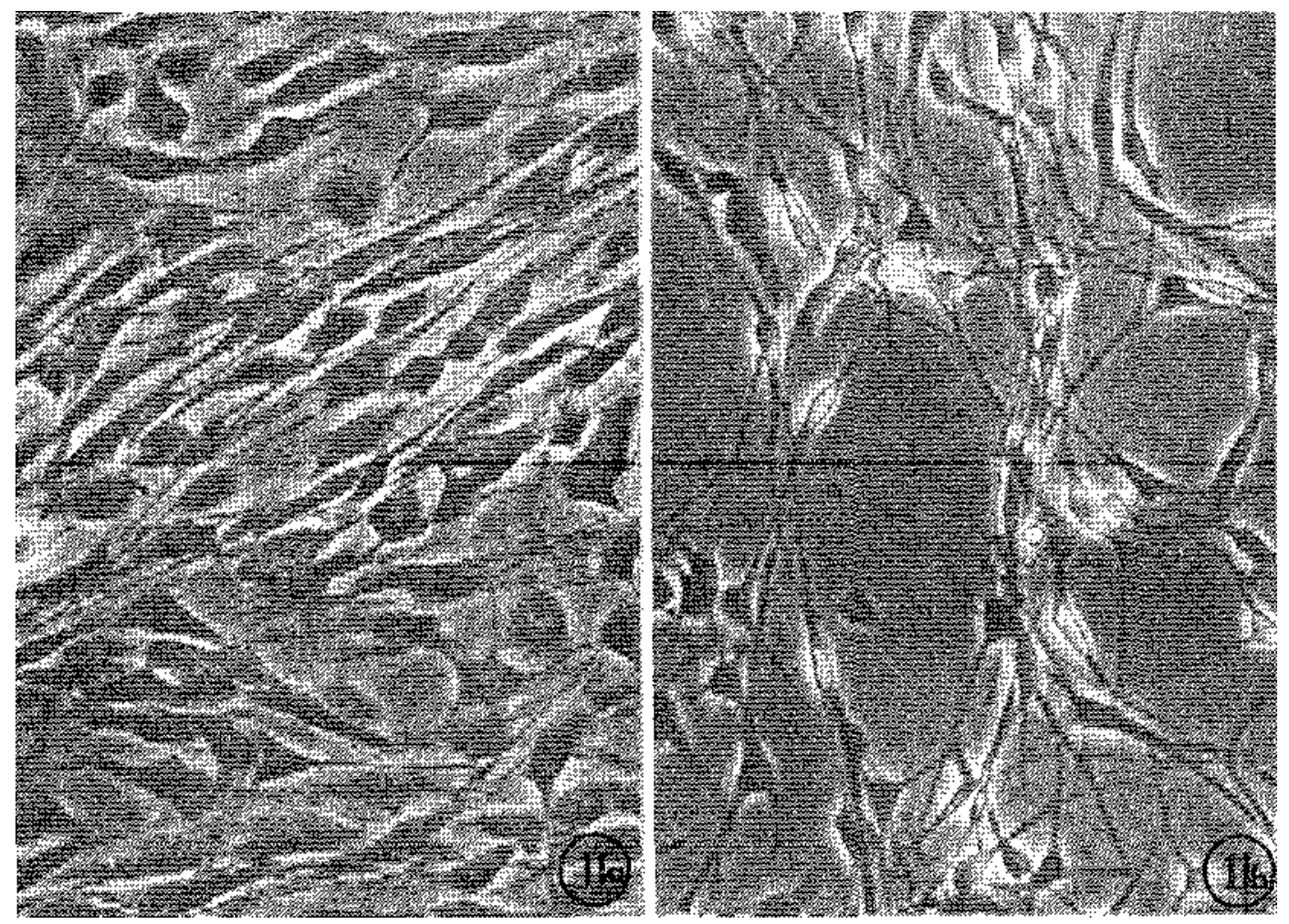
Fig. 12. SEM of secondary SC 4 days after removing mitogenic factors. Recovery of spindle shape is partial. The flat cells correspond to cholera toxin modified cells as shown in fig.11a.. During these cell shape changes, more glass surface becomes visible (G). X510.

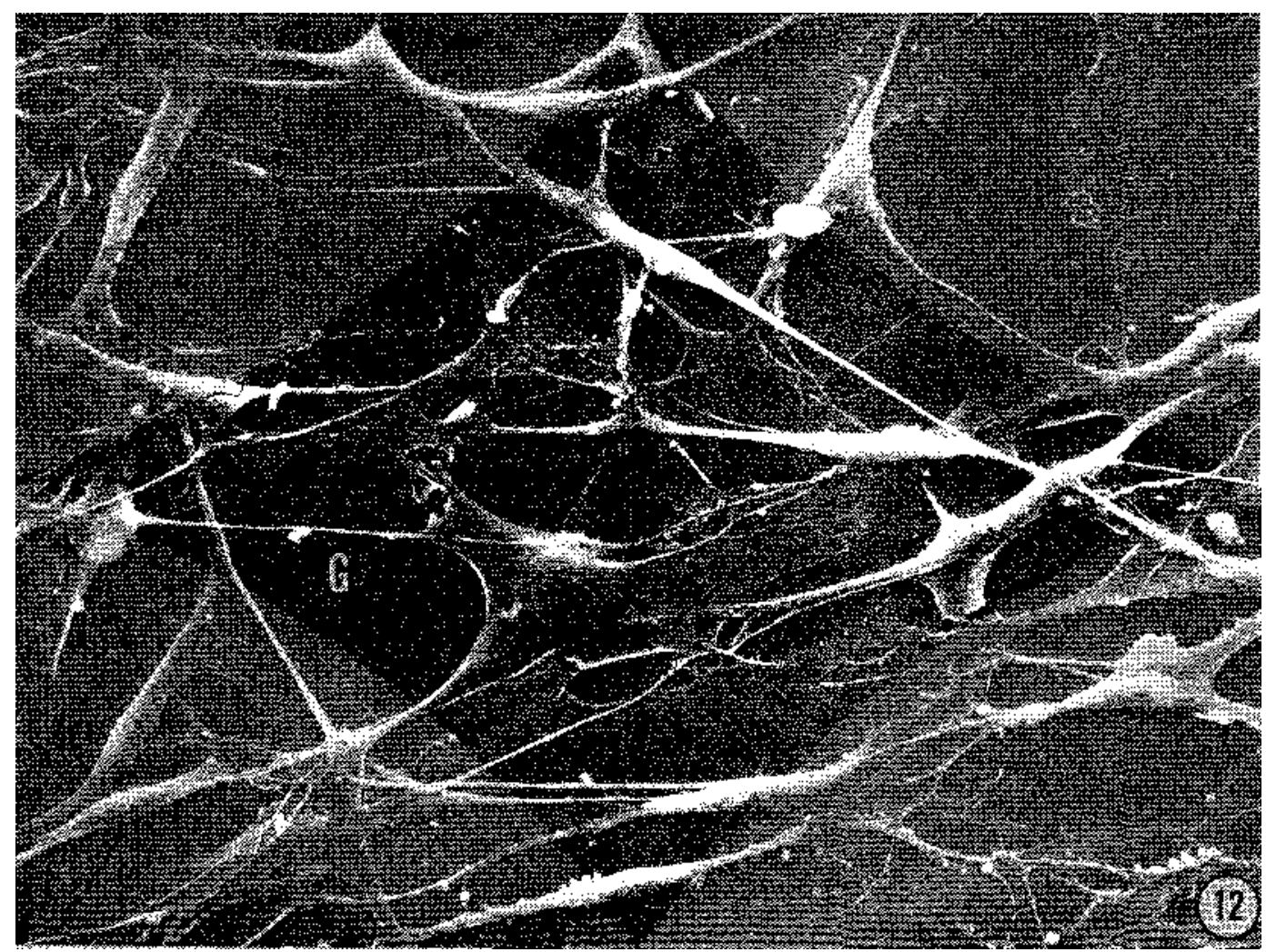

\section{DISCUSSION}

In this study we have analyzed the motility and structure of SC in isolation shortly after sciatic nerve dissociation, as well as after intense mitotic stimulation and multiple subcultures. Our observations revealed that secondary SC, which became stable after numerous mitoses, recovered most of their original characteristics. In addition, these secondary SC, as well as primary SC, resemble some of the SC observed in vivo, whether migrating, reactive, or dedifferentiated $[15,25,26]$.

Many of the sciatic nerve cells from newborn rats are myelin-related [27], hence the nerve dissociation creates intense injury with disintegration of fibers undergoing the process of myelination. This might trigger the mitoses observed early in primary SC cultures (15-30\% according to [3]), since direct mechanical injury during degeneration is a mitotic stimulant for SC [27]. Once dissociated and separated from neurites, SC still display longitudinal extension of their cytoplasm. The latter contains myelin inclusions which progressively disappear. The fastest digestion of the myelin components occurs in high glucose medium with serum, the slowest in serum-free medium.

Stable primary and secondary SC have remarkable undulatory activities and intense migratory activities completely distinct from the apparently erratic fibroblast migration. The undulatory activity of primary SC has been observed by several authors [20-23, 38]. Most of them have noticed that the duration of undulation is relatively constant and that the resting period between undulations is highly variable from cell to cell, and for one single cell. Our long-term observations have revealed that even though the periodicity is variable, the total number of cycles/day in chemically defined medium is rigorously constant. This indicates that the moment of undulation is determined by variable conditions in the culture (attachment of the cell by one or two processes, contacts with other cells, etc.), but the overall uniformity probably reflects a genotypic basis. No synchronism between neighboring cells could ever be detected. The possible relationship between pulsatile activity observed in vitro and specific function of SC in vivo has been discussed in detail [38]. The pulsation could play a role in (1) putative spiraling of Schwann cells around axons during myelination; (2) withdrawal of cytoplasm resulting in the major dense line of myelin; (3) circulation of cytoplasmic material in myelin [28].

Migratory activity of isolated SC results from the combination of process extension and retraction, and nuclear movement within the cell $[21,22]$. Migration is oriented in a polar fashion, perhaps a reminder of the oriented movement of SC along axons in vivo. Interestingly, migratory SC in the developing nerve lack basal lamina as in 
our cultured SC [15]. It has been shown both in vivo and in vitro that SC acquire a basal lamina only when they become associated with intact neurites $[15,18]$. SC also show numerous actin and intermediate filaments; actin is necessary to movement, and intermediate filaments have a crucial role in regulation of cell shape [29]. Filaments have also been described in actively migrating glial cells [30]. Generally, the advance of a cell body is associated with filament bundles which act as skeletal organelles. Although prominent filaments are not a characteristic of resting SC in vivo, the presence of longitudinal filaments has been noted in SC processes during mitosis in newborn rat sciatic nerve [31] and in undifferentiated SC which proliferate in nerve stumps after chronic denervation [26]. It has been suggested that SC indefinitely preserve their potential of migration even when they are fixed in the adult nerve. Migration resumes if nerve degeneration occurs [32].

The mitogenic effect resulting from synergy between PITF, CT and FCS [5] is three times higher than that observed with neurite membrane fraction from sensory neurons [33, 34]. The combined use of PITF and CT, has resulted in the accumulation of millions of cells with CT phenotype. These cells are multipolar and flat, and contain a prominent Golgi and numerous ribo-somes as do the "reactive" SC observed in vivo after nerve crush [25]. They also display specific pulsations which are very different from the undulations of the stable cells although the periodicity appears to be maintained with the same frequency. For this reason, we believe that the characteristic pulsation of secondary SC corresponds to the undulation of primary SC, its appearance being different because of mode of attachment to the substrate. Secondary SC are flat and attached over their entire surface, whereas primary SC are elongated and attached by their extremities only. Secondary SC thus cannot undulate and the contractions appear as pulsations. Influence of the mode of attachment on undulation has been noted previously [21]. The mobility of secondary SC during their growth phase is greatly reduced compared to primary SC. Their migratory speed is 5 times slower. This is probably also related to their mode of attachment.

After intense mitotic stimulation, SC will progressively acquire a typical primary SC morphology, while they recover an undulating movement, gradually replacing the secondary SC pulsation. Although the frequency of pulsation is doubled shortly after removal of mitotic factors, it eventually resumes the primary SC periodicity. The migration speed increases up to $2 / 3$ of the primary SC speed. Perhaps the artificial mitotic stimulation in vitro produces "naive" SC which, under axonal influence, might be able to specialize and redifferentiate. Similarly, in vivo, SC undergo mitosis before reacquiring developmental multi-potentiality after nerve injury [35]. Whether secondary SC will be able to synthesize organized myelin around axons is presently under investigation. It is hoped that structural and motility studies of the kind presented here could be performed on human Schwann cells which have been successfully cultured from nerve expiants in early and late studies [36, 37].

\section{ACKNOWLEDGEMENTS}

We wish to thank Drs J. Brockes, M. Raff and B. Trapp for precious advise throughout this study and the gift of antisera, Dr D. Forman for fruitful discussions of SC motility, Ray Rusten for skillful technical help, Ann Claysmith for review of the manuscript and Lin Aspinall for typing. B.R. is visiting associate from the Fogarty International Center.

\section{REFERENCES}

1. Wood, P M \& Bunge, R P, Nature 256 (1975) 662.

2. Wood, PM, Brain res 115(1976)361.

3. Brockes, J P, Fields, K L \& Raff. M C, Brain res 165 (1979) 105.

4. - Nature 266 (1977) 364.

5. Raff, M C, Abney, E, Brockes, J P \& Hornby-Smith, A, Cell 15 (1978) 813.

6. Mirsky, R, Winter, J, Abney, E R, Pruss, R M, Gavrilovic, J \& Raff, M C, J cell biol 84 (1980) 483.

7. Brockes, J P, Raff, M C, Nishiguishi, D J \& Winter, J, J neurocytol 9 (1980) 67

8. Willingham, M C \& Pastan, I, Cell 13 (1978) 501.

9. Faulkner, G, Dubois-Dalcq, M, Hooghe-Peters, E, McFarland, H \& Lazzarini, R A, Cell 17 (1979) 979.

10. Bottenstein, J E \& Sato, G H, Proc natl acad sci US 76 (1979) 514.

11. Raff, M C, Hornby-Smith, A \& Brockes, J, Nature 273 (1978) 672.

12. Brockes, J P, Lemke, G E \& Balzer, D R, J biol chem 225 (1980) 8374.

13. Fields, K L, Brockes, J P, Mirsky, R \& Wendon, L MB, Cell 14(1978)43. 
Published in: Experimental cell research (1981), vol. 131, iss. 2, pp. 283-297

Status: Postprint (Author's version)

14. Trapp, B D, Mclntyre, L J, Quarles, R H, Sternberger, N H \& Webster, H de F, Proc natl acad sci US 76 (1979) 3552.

15. Webster, H de F, Peripheral neuropathy (ed. P J Dyck, P K Thomas \& W B Lambert) Saunders Co. (1975) 37.

16. Odenwald, W F, Askanas, V, Engel, W K, Carter, L S \& Lawrence, J V, Soc for neurosci, abstr. 5 (1979) 756.

17. Bunge, M, Williams, A K \& Wood, P M, Cell biol. Abstr. 19th ann. meet. Am soc cell biol 83 (1979) 130a.

18. Bunge, M B, Williams, A K, Wood, P M, Uitto, J \& Jeffrey. J J, J cell biol 84 (1980) 184.

19. Middleton, C A, Nature 282 (1979) 203.

20. Pomerat, C M, Science 130 (1959) 1759.

21. Ernyei, S \& Young, M R, J physiol 183 (1966) 469.

22. Cravioto, H \& Lockwood. R, Z Zellforsch 90 (1968) 186.

23. Forman. D S, Shain, W G, Fuchs, D A \& Brad-dock, C H, Soc neurosci, abstr. 5 (1979) 755.

24. Brockes, J P \& Raff, M C, In vitro 15 (1979) 772.

25. Bray, G M, Peyronnard, J M \& Aguayo, A J, Brain res 42 (1972) 297.

26. Spencer, P S, Weinberg, H J, Krygier-Brevart, V \& Zabrenetzky, V, Brain res 165 (1979) 119.

27. Salzer, J L \& Bunge, R P, J cell biol 84 (1980) 739.

28. Singer, M \& Bryant, S V, Nature 221 (1969) 1148.

29. Lazarides, E, Nature 283 (1980) 249.

30. Ludueña, M A \& Wessells, N K, Dev biol 30 (1973) 427

31. Martin, J R \& Webster, H de F, Dev biol 32 (1973) 417.

32. Lubinska, L, Exp cell res suppl. 8 (1961) 74.

33. Salzer, J L, Williams, A K, Glaser, L \& Bunge, R P, J cell biol 84 (1980) 753.

34. Salzer, J L, Bunge, R P \& Glazer, L, J cell biol 84 (1980) 767.

35. Aguayo, A, Epps, J, Charron, L \& Bray, J M, Brain res 104(1976) 1.

36. Murray, M R \& Stout, A P, Anat rec 84 (1942) 275.

37. Askanas, V, Engel, W K, Dalakas, M, Lawrence, J V \& Carter, L F, Arch neurol 37 (1980) 329.

38. Forman et al. Submitted for publication. 